\section{Características clínicas \\ e epidemiológicas da \\ leishmaniose visceral em \\ crianças internadas em um \\ hospital universitário de \\ referência no norte de Minas \\ Gerais, Brasil}

\author{
Clinical and epidemiological \\ characteristics of visceral \\ leishmaniasis in children
}

hospitalized at a reference university hospital in the north of Minas Gerais, Brazil

\section{Ludmila Mourão Xavier-Gomes'}

Wagner Barreto Costa'

Patrícia Fernandes do Prado'

Maryane Oliveira-Campos"

\section{Maisa Tavares de Souza Leite'}

"Departamento de Enfermagem da Universidade Estadual de Montes Claros, MG - UNIMONTES.

"Escola de Enfermagem da Universidade Federal de Minas Gerais - UFMG.

\section{Resumo}

Objetivo: Descrever as características clínicas e epidemiológicas e o tratamento das crianças internadas com leishmaniose visceral (LV) em hospital universitário de referência no norte de Minas Gerais, Brasil. Métodos: Estudo retrospectivo e documental de crianças de 0 a 12 anos internadas com diagnóstico de LV no Hospital Universitário Clemente de Faria, Montes Claros, MG. Foram analisados os prontuários referentes ao período de janeiro de 2006 a dezembro de 2007. Resultados: Foram identificadas 51 crianças com $\mathrm{LV}$, sendo $51 \%$ do sexo feminino e a faixa etária de maior incidência da doença foi em menores de 5 anos (74,5\% dos casos). Verificou-se que $31 \%$ dessas crianças residiam em Montes Claros e $69 \%$ procediam de 20 municípios do norte de Minas Gerais, dos quais 72,5\% eram originárias da zona urbana e $21,6 \%$ da zona rural. A principal manifestação clínica foi a febre $(96,1 \%)$ e os principais achados clínicos na admissão foram esplenomegalia (98\%) e hepatomegalia (94\%). O tratamento de escolha foi Glucantime (70,6\%), Anfotericina B convencional (13,7\%), Anfotericina B lipossomal (2\%) e Glucantime associado a Anfotericina B (15,7\%). 35,4\% dos casos desenvolveram processos infecciosos durante o período da internação, destacando-se as infecções de pele em $11,8 \%$. O tempo de permanência hospitalar médio foi de 19 dias $(\mathrm{DP}= \pm 5,4), 96,1 \%$ receberam alta médica e 3,9\% evoluíram para óbito. Conclusões: A partir das características clínicas e epidemiológicas identificadas no estudo, sugere-se uma observação mais eficaz por parte dos profissionais de saúde, visando ao reconhecimento precoce e tratamento adequado da doença e suas complicações.

Palavras-chave: Leishmaniose. Epidemiologia. Criança.

O presente trabalho encontra-se vinculado à Universidade Estadual de Montes Claros - Unimontes. Correspondência: Ludmila Mourão Xavier Gomes. Universidade Estadual de Montes Claros - Unimontes - Departamento de Enfermagem. Av. Francisco Gaetani, 673, Major Prates, Montes Claros, MG CEP: 39403-202. Email: ludyxavier@yahoo.com.br 


\section{Abstract}

Objective: To describe the clinical and epidemiological characteristics and treatment of children admitted with visceral leishmaniasis (VL) to a reference university hospital in the northern part of Minas Gerais, Brazil. Methods: Retrospective study and documentation of children aged 0 to 12 years admitted with a diagnosis of VL at the University Hospital Clemente de Faria, Montes Claros, MG. Records for the period between January 2006 and December 2007 were analyzed. Results: We identified 51 children with VL, of which $51 \%$ were female, and $74.5 \%$ were under 5 years of age. Children came from Montes Claros (31\%), 20 municipalities in the north of the state of Minas Gerais (69\%), urban areas $(72.5 \%)$, and rural areas (21.6\%). The main clinical manifestation was fever (96.1\%) and major clinical findings at admission were splenomegaly (98\%) and hepatomegaly (94\%). The treatment of choice was Glucantime (70.6\%), Conventional Amphotericin B (13.7\%), Liposomal Amphotericin B (2\%), and Glucantime associated with Amphotericin B (15.7\%). Infections developed during hospitalization in $35.4 \%$ of cases, and of those, $11.8 \%$ were skin infections. The average length of hospitalization was 19 days $(\mathrm{SD}= \pm 5.4$ ); $96.1 \%$ children received medical discharge and $3.9 \%$ progressed to death. Conclusions: From the clinical and epidemiological characteristics identified in the study, more effective monitoring by healthcare workers is recommended, aiming at early recognition and appropriate treatment of the disease and its complications.

Keywords: Leishmaniasis. Epidemiology. Child.

\section{Introdução}

A leishmaniose visceral (LV) é uma parasitose endêmica das Américas que está entre as sete endemias de prioridade da Organização Mundial de Saúde ${ }^{1}$. Estima-se que sua incidência seja de 500.000 casos novos e 50.000 mortes a cada ano no mundo, com números em ascensão ${ }^{2}$. Nas Américas, o Brasil representa o país de maior endemia, responsável por aproximadamente $97 \%$ de todos os casos no continente ${ }^{3}$. A doença apresenta casos autóctones notificados em pelo menos 19 Estados da federação brasileira, distribuídos em quatro das cinco regiões, permanecendo indene apenas o $\mathrm{Sul}^{4}$.

É uma doença sistêmica causada por um protozoário do gênero Leishmania, que se reproduz dentro do sistema fagocítico mononuclear de hospedeiros mamíferos suscetíveis. No Brasil, o principal vetor é o Lutzomya longipalpis. No ambiente domiciliar, o cão doméstico é o reservatório mais importante, e o homem é o hospedeiro final ${ }^{4}$.

Uma importante mudança no padrão epidemiológico da LV tem sido verificada: caracterizada como uma doença rural, vem apresentando um processo de urbanização com crescente expansão para cidades de médio e grande porte, principalmente nas regiões sudeste e centro-oeste ${ }^{5}$.

A suscetibilidade é universal, atingindo pessoas de todas as idades e sexo. Entretanto, no Brasil, a doença atinge principalmente a população infantil, predominantemente nos seis primeiros anos de vida ${ }^{3}$. A importância da LV em nosso país reside na sua alta incidência, na ampla distribuição e no seu potencial de assumir formas graves e letais quando associada aos quadros de desnutrição e infecções concomitantes ${ }^{4}$.

O Hospital Universitário Clemente de Faria (HUCF) é o único hospital público de referência para o Sistema Único de Saúde no tratamento de $\mathrm{LV}$ atendendo pacientes procedentes do norte de Minas Gerais. Neste contexto, foi proposto como objetivo deste estudo descrever as características clínicas e epidemiológicas das crianças internadas com diagnóstico de LV. 


\section{Material e métodos}

Trata-se de um estudo exploratório, descritivo e transversal de abordagem quantitativa. A pesquisa foi submetida à apreciação do Comitê de Ética e Pesquisa da Universidade Estadual de Montes Claros, aprovado por meio do Parecer Consubstanciado $n^{\circ} 745 / 07$.

Foram estudados os dados de 51 pacientes de zero a 12 anos de idade internados com diagnóstico de LV no HUCF no período de janeiro de 2006 a dezembro de 2007. Os dados foram obtidos por meio dos prontuários disponibilizados pelo Serviço de Arquivo Médico e de Estatística e das fichas de notificação compulsória do Núcleo de Vigilância Epidemiológica do HUCF. O instrumento de coleta de dados foi elaborado pelos pesquisadores por meio de um formulário padronizado, com as seguintes variáveis: idade, sexo, tipo de entrada, procedência, infraestrutura básica, sintomas referidos pelo responsável, dados clínicos, exames laboratoriais e tratamento.

Para a interpretação dos dados, a leucopenia foi considerada quando a contagem de leucócitos foi abaixo de $5.000 / \mathrm{mm}^{3}$, e a plaquetopenia quando a contagem de plaquetas foi inferior a $150.000 / \mathrm{mm}^{3}$. A anemia foi definida como um valor de hemoglobina $<11 \mathrm{~g} / \mathrm{dl}$ para pacientes entre 6 meses e 5 anos, $<11,5 \mathrm{~g} / \mathrm{dl}$ entre 5 a 9 anos, $<12 \mathrm{~g} / \mathrm{dl}$ para adolescentes femininos $\mathrm{e}<12,5 \mathrm{~g} / \mathrm{dl}$ para adolescentes masculinos ${ }^{6}$.

Os dados foram consolidados a partir da utilização do Programa Statistical Package of Social of Science (SPSS) versão 13.0, que permitiu a análise estatística descritiva do estudo.

\section{Resultados}

Foram analisados os dados de 51 pacientes, 17 (33,3\%) internados em 2006 e 34 $(66,7 \%)$ em 2007 , dos quais $47(92,2 \%)$ eram casos de primoinfecção e $4(7,8 \%)$ de recidiva. A faixa etária de maior incidência da $\mathrm{LV}$ foi a de menor ou igual a 5 anos $(78,4 \%)$ e $51 \%$ das crianças eram do sexo feminino (Tabela 1).
Dentre as características do domicílio, a ausência de água encanada e a de esgotamento sanitário foram observadas em 23,5\% e $31,4 \%$, respectivamente. Verificando a cidade de origem na ocasião do adoecimento, observou-se que $31 \%$ das crianças residiam em Montes Claros e $69 \%$ procediam de outros 20 municípios do norte de Minas Gerais. Além disso, constatou-se que $72,5 \%$ dos casos eram originários da zona urbana, 21,6\% da zona rural e 5,9\% não apresentaram esta informação nos prontuários.

Dentre as manifestações clínicas apresentadas pelas crianças do estudo, a febre foi o maior motivo de procura de atendimento por parte dos responsáveis $(96,1 \%$ dos casos). Além da febre, o aumento do volume abdominal (49\%) e a hiporexia $(33,3 \%)$ foram os sintomas mais frequentes, seguidos de tosse $(21,6 \%)$, palidez $(15,7 \%)$, dor abdominal $(17,6 \%)$, emagrecimento $(19,6 \%)$ e astenia $(11,8 \%)$. Os principais achados do exame clínico na admissão foram esplenomegalia em $98 \%$ dos casos, hepatomegalia $(94,1 \%)$, palidez cutânea (2\%), edema $(15,7 \%)$, icterícia $(3.9 \%)$ e dispnéia (2\%).

Em relação aos dados hematológicos no momento da admissão, o nível médio de hemoglobina foi de $7,12 \mathrm{~g} / \mathrm{dl}$, variando de 2,30 a 12,80 g/dl. A contagem média de leucócitos foi de $4.668 / \mathrm{mm}^{3}$, variando de 1.100 a $17.000 / \mathrm{mm}^{3}$. A mediana da contagem absoluta de plaquetas foi de $82.578 /$ $\mathrm{mm}^{3}$, variando de 14.000 a $274.000 / \mathrm{mm}^{3}$.

Verificou-se que a microscopia direta do aspirado medular foi realizada em 36 pacientes, porém só foi possível obter a informação sobre o resultado em 25 casos, dos quais se constatou que $56 \%$ foram considerados positivos e $44 \%$ negativos. O método de Reação de imunofluorescência indireta (RIFI), considerado positivo para títulos acima de 80 , foi realizado em $34(67 \%)$ casos, dos quais $59 \%$ foram positivos e $41 \%$ negativos. O teste da fita com antígeno rK39, conhecido como Teste Rápido Anticorpo Anti - Leishmania donovani - TRALd, foi realizado em 48 (94\%) dos casos pesquisados. $100 \%$ foram considerados positivos. 
Tabela 1 - Características sociodemográficas das crianças internadas com diagnóstico de leishmaniose visceral no HUCF, no período de janeiro de 2006 a dezembro de 2007.

Table 1 - Socio-demographic characteristics of children admitted with a diagnosis of visceral leishmaniasis to HUCF, from January 2006 to December 2007.

\begin{tabular}{|c|c|c|}
\hline Características sociodemográficas & $\mathrm{n}$ & $\%$ \\
\hline \multicolumn{3}{|l|}{ Tipo de entrada } \\
\hline Primoinfecção & 47 & 92,2 \\
\hline Recidiva & 4 & 7,8 \\
\hline \multicolumn{3}{|l|}{ Sexo } \\
\hline Feminino & 26 & 51,0 \\
\hline Masculino & 25 & 49,0 \\
\hline \multicolumn{3}{|l|}{ Idade } \\
\hline$\leq 5$ anos & 40 & 78,4 \\
\hline$>5$ anos & 11 & 21,6 \\
\hline \multicolumn{3}{|l|}{ Procedência } \\
\hline Zona Urbana & 37 & 72,5 \\
\hline Zona Rural & 11 & 21,6 \\
\hline Não informado & 3 & 5,9 \\
\hline \multicolumn{3}{|l|}{ Infraestrutura básica } \\
\hline \multicolumn{3}{|l|}{ Esgotamento sanitário } \\
\hline Sim & 31 & 60,8 \\
\hline Não & 16 & 31,4 \\
\hline Não informado & 4 & 7,8 \\
\hline \multicolumn{3}{|l|}{ Água encanada } \\
\hline Sim & 34 & 66,7 \\
\hline Não & 12 & 23,5 \\
\hline Não informado & 5 & 9,8 \\
\hline
\end{tabular}

Nota: Os valores absolutos e percentuais de cada variável da tabela totalizam, respectivamente, 51 e 100\%.

Note: Absolute values and percentages of each variable inf the table are 51 and 100\% respectively.

O tratamento de escolha para a LV foi o Antimoniato de N-metil glucamina (Glucantime) em 70,6\% dos casos, sendo que um desses pacientes apresentou cardiotoxicidade à droga, empregando-se a Anfotericina B lipossomal como segunda escolha. A Anfotericina B convencional foi utilizada como primeira escolha em $13,7 \%$ dos casos. Utilizou-se o Glucantime associado com a Anfotericina B em 15,7\% das crianças hospitalizadas.

Durante o período de internação $35,4 \%$ desenvolveram processos infecciosos. Dentre estes, as infecções de pele se destacaram em $11,8 \%$, seguidas das infecções do trato urinário (9,8\%), pneumonia $(7,8 \%)$, infecção da cavidade oral (2\%), sepse ( $2 \%$ ) e infecções com foco não identificado (2\%). Além disso, verificou-se que um dos pacientes apresentou hemorragia intestinal e pulmonar, evoluindo com septicemia e óbito.

O tempo de permanência hospitalar médio foi de 19 dias $(\mathrm{DP}= \pm 5,4)$. Quanto à evolução dos casos, 96,1\% receberam alta médica e 3,9\% evoluíram para óbito.

\section{Discussão}

A frequência da doença em menores de 5 anos verificada no presente estudo também foi observada nos estudos de Cardoso ${ }^{7}$, em que $78 \%$ das crianças com LV tinham menos 
que 6 anos de idade, e de Brustolini ${ }^{5}$, em que a doença predominou em $69,9 \%$ nos primeiros cinco anos de vida.

Nesta série, crianças do sexo masculino e feminino foram igualmente afetadas pela doença, discordando da maioria dos achados de literatura que aponta o sexo masculino como mais susceptível ao adoecimento $^{5,8,9}$, razão ainda não totalmente esclarecida, postulando-se a existência de um fator hormonal ligado ao sexo ou à exposição ${ }^{10}$.

O número de municípios com registros de casos de LV confirma que, a exemplo de outras localidades brasileiras, é uma doença em expansão no Norte de Minas e também vem apresentando uma forte tendência à urbanização, fato este já destacado por diversos autores ${ }^{4,9,11}$.

Segundo Monteiro et al. ${ }^{12}$, no município de Montes Claros é encontrado um ambiente característico e propício à ocorrência de LV. As habitações são, em sua maioria, extremamente pobres, com deficiência na coleta de lixo e no saneamento básico. Em algumas áreas, muitos moradores possuem baixos índices socioeconômicos e a convivência com animais domésticos é bastante elevada, resultando em acúmulo de matéria orgânica e proporcionando condições favoráveis para a ocorrência da transmissão da doença. Esses aspectos também são citados nos estudos de Sherlock ${ }^{13}$, que observou, na Bahia e em outras regiões do país, que pobreza, desnutrição, grande número de cães infectados, além da alta densidade de flebotomíneos tanto no intradomicílio como no peridomicílio, estão associados ao grande número de animais doméstico, péssimas condições sanitárias e baixo nível socioeconômico.

As manifestações clínicas apresentadas pelas crianças do estudo não diferiram das classicamente encontradas nas literaturas, tanto para os sintomas referidos pelos acompanhantes quanto para os sinais físicos na admissão. Em nossa amostra, a febre foi o maior motivo de procura de atendimento médico por parte dos responsáveis, dados semelhantes aos encontrados por
Pedrosa, da Rocha ${ }^{14}$, Rey et al..$^{15}$ e Oliveira et $\mathrm{al}^{16}$.

Os achados do exame clínico na admissão mostraram que o aumento do fígado e do baço e a palidez cutânea foram os sinais mais frequentes, também relatados em outros estudos ${ }^{3,6,17}$.

A análise do hemograma evidenciou que, na admissão, 98\% das crianças apresentavam anemia, $96 \%$ plaquetopenia e $74 \%$ leucopenia. Achados semelhantes foram documentados em outros estudos com crianças acometidas pela LV por Cardoso ${ }^{7}$, o qual mostrou que, na admissão, $100 \%$ das crianças apresentavam anemia, $96 \%$ plaquetopenia e $78 \%$ leucopenia, e por Brustolini ${ }^{5}$, segundo o qual a anemia foi constatada em mais de $90 \%$ dos casos de crianças com calazar, mais de $90 \%$ apresentavam plaquetopenia e $80 \%$ mostravam leucopenia.

Em relação ao diagnóstico parasitológico, deve-se levar em consideração que mais de $31 \%$ dos resultados do aspirado medular não estavam registrados nos prontuários dos casos estudados, fator esse que demonstra a necessidade de uma maior valorização dos registros por parte dos profissionais de saúde, viabilizando dados para futuras pesquisas e, conseqüentemente, a obtenção de resultados mais fidedignos. No Brasil, a positividade da microscopia direta em pacientes pediátricos tem sido relatada variando de $69,3 \%$ a $81,2 \%{ }^{6}$.

Dentre os testes sorológicos realizados, verificou-se que, em 17 casos, não foram encontrados registros referentes ao uso da RIFI, reação sorológica mais utilizada para o diagnóstico da $\mathrm{LV}^{18}$, cuja sensibilidade varia de $55 \%$ a $96 \%$ e a especificidade é relatada em torno de $70 \%$ a $98 \%{ }^{19}$. Os resultados encontrados no uso do TRALd, abordado por alguns autores como o mais potente e promissor teste sorológico ${ }^{20}$, foram semelhantes aos encontrados em outras literaturas, alcançando sensibilidade de $95 \%$ a $100 \%$.

Os processos infecciosos identificados refletem a suscetibilidade da criança às infecções ${ }^{21}$. Para Santos et al. ${ }^{20}$, a coinfecção bacteriana está associada à imunodepressão causada pela $\mathrm{LV}$, pois quanto menor 
for a resposta celular do paciente, menor será a resposta à terapêutica específica e maior será o surgimento de infecções oportunistas.

$\mathrm{O}$ antimonial pentavalente foi a droga mais utilizada no tratamento das crianças do presente estudo e continua a ser a droga mais adequada para a utilização em crianças, pois causa efeitos adversos de pouca gravidade, geralmente reversíveis, e é muito eficaz ${ }^{5}$.

\section{Conclusão}

Os resultados obtidos sugerem que a LV está amplamente distribuída no norte de Minas Gerais, caracterizando a região como importante área endêmica. Assim, a produção de dados ou de informações sobre as características clínicas e epidemiológicas identificadas no estudo sugere uma observação mais eficaz por parte dos profissionais de saúde, visando ao reconhecimento precoce e ao tratamento adequado da doença e de suas complicações, no intuito de diminuir as estatísticas de morbidade e a hospitalização infantil por LV.

Além disso, os resultados encontrados impõem aos municípios atingidos pela doença a sua análise epidemiológica em suas áreas de ocorrência, a fim de que medidas de controle apropriadas possam ser rapidamente efetivadas pelos órgãos competentes. Dentre essas medidas, recomenda-se: busca ativa de doentes e encaminhamento para diagnóstico e tratamento; inquérito sorológico canino; apreensão e eliminação sumária dos cães soropositivos; borrifação sistemática de inseticida residual nos domicílios e peridomicílios; e programas de educação comunitária4 .

Deve haver também uma capacitação dos profissionais de saúde para sensibilizar a comunidade sobre a importância da preservação do meio-ambiente e sobre as formas de se evitar a proliferação dos vetores, visando a diminuir a transmissão da LV nestas regiões.

\section{Referências}

1. Duarte MIS. Leishmaniose visceral. In: Filho GB. Patologia. $6^{\text {a }}$ ed. Rio de Janeiro: Guanabara Koogan; 2000. p. 1215-1227.

2. Desjeux P. Leishmaniasis: current situation and new perspectives. Comp Immunol Microbiol Infect Dis 2004; 27: 305-18.

3. Pastorino AC, Jacob CMA, Oselka G, Carneiro-Sampaio MMS. Leishmaniose visceral: aspectos clínicos e laboratoriais. J Pediatr 2002; 78(2): 120-7.

4. Brasil, Ministério da Saúde. Secretaria de Vigilância em Saúde. Manual de Vigilância e controle da Leishmaniose visceral. Brasília, DF: Ministério da Saúde; 2006.

5. Brustoloni YM. Leishmaniose visceral em crianças no Estado de Mato Grosso do Sul, Brasil: contribuição ao diagnóstico e ao tratamento [tese de doutorado]. Campo Grande: Universidade Federal de Mato Grosso do Sul; 2006.

6. Queiroz MJA, Alves JGB, Correia JB. Leishmaniose visceral: características clínico-epidemiológicas em crianças de área endêmica. J Pediatr 2004; 80: 141-6.

7. Cardoso VV. Manifestações clínicas, laboratoriais, e a função dos fagócitos em crianças com leishmaniose visceral tratadas com glucantime [dissertação de mestrado]. Brasília: Universidade de Brasília; 2007.
8. Correa JB. Epidemioly of Visceral Leishmaniasis in Pernambuco, Northeast of Brazil and the use of a Latex Agglutination Test in Urine for its Diagnosis [thesis for the Degree of Master of Tropical Pediátricas] Liverpool School of Tropical Medicine: Liverpool; 1998.

9. Brasil. Ministério Nacional de Saúde. Fundação Nacional de Saúde (FUNASA). Controle, diagnóstico e tratamento da leishmaniose visceral (calazar): Normas Técnicas. Brasília: Ministério Nacional da Saúde; 1999.

10. Costa HNC, Pereira HF, Araújo MV. Epidemia de leishmaniose visceral no estado do Piauí, Brasil 19801986. Rev Saúde Pública 1990; 24(5): 361-72.

11. Miranda, Gabriella Morais Duarte. Leishmaniose visceral em Pernambuco: a influência da urbanização e da desigualdade social. [dissertação de mestrado]. Recife: Fundação Oswaldo Cruz; 2008.

12. Monteiro EM, França-Silva JC, Costa RT, Costa DC, Barata RA, Paula EV et al. Leishmaniose visceral: estudo de flebotomíneos e infecção canina em Montes Claros, Minas Gerais. Rev Soc Bras Med Trop 2005; 38 (2): 147-52.

13. Sherlock IA. Ecological interactions of visceral leishmaniasis in the State of Bahia. Memórias do Instituto Oswaldo Cruz 1996; 91: 671- 83. 
14. Pedrosa CM, Da Rocha EM. Aspectos clínicoepidemológicos da leishmaniose visceral em menores de 15 anos procedentes de Alagoas, Brasil. Rev Soc Bras Med Trop 2004; 37(4): 300-4.

15. Rey LC, Martins CV, Ribeiro HB, Lima AAM. American visceral leishmaniasis (kalazar) in hospitalized children from an endemic area. J Pediatr 2005; 81: 73-84.

16. Oliveira AL, Paniago AMM, Dorval MEC, Oshiro ET, Leal CR, Sanches M, et al et al. Foco emergente de leishmaniose visceral em Mato Grosso do Sul. Rev Soc Bras Med Trop 2006; 39(5): 446-50.

17. Silva AO, Silva PB, Silva OV, Melo AA, Leite JA, Pinheiro AJ, et al. Leishmaniose visceral no agreste Pernambucano: casos humanos. Rev Soc Bras Med Trop 2001; 34(S1): 224.

18. Santos MA, Marques RC, Farias CA, Vasconcelos DM. Indicadores de reposta insatisfatória ao antimônio pentavalente no tratamento da leishmaniose visceral americana. Rev Soc Bras Med Trop 2002; 35: 629-33.
19. Tamayo, CO. Avaliação da co-infecção por Leishmania em pessoas vivendo com HIVIAIDS acompanhadas no Hospital Universitário de Brasília [dissertação de mestrado]. Brasília: Universidade de Brasília; 2006.

20. Alves WA, Bevilacqua PD. Reflexões sobre a qualidade do diagnóstico da leishmaniose visceral canina em inquéritos epidemiológicos: o caso da epidemia de Belo Horizonte, Minas Gerais, Brasil, 1993-1997. Cad Saúde Pública 2004; 20 (1): 259-65.

21. Cladas AJ, Costa J, Aquino D, Silva AA, Barral A. Are there differences in clinical and laboratory parameters between children and adults with American visceral leishmaniases? Acta Trop 2006; 97 (3): 525-8.

Recebido em: 27/01/09 Versão final reapresentada em: 14/08/09 Aprovado em: 24/08/09 